\title{
Dagmar Yu-Dembski
}

\section{Frauen in China Transformation und sozialer Wandel}

Seit den 80er Jahren findet in China ein tiefgreifender gesellschaftlicher Umstrukturierungsprozeß statt, der durch die Umwandlung des planwirtschaftlichen Systems in eine marktorientierte Ökonomie gekennzeichnet ist. Gleichzeitig mit der wirtschaftlichen Umgestaltung der als sozialistisch apostrophierten Gesellschaft wird die Dezentralisierung und Industrialisierung des Agrarlandes vorangetrieben. Die ökonomische Reform und „Öffnung“ des Landes ist verknüpft mit der Einbindung Chinas in die Mechanismen und Strukturen des globalen Marktes; dieser Prozeß ist charakterisiert durch ausländische Kapitalinvestitionen in den Sonderwirtschaftsgebieten, Exportorientierung und Bereitstellung billiger Arbeitskräfte. Der Kurs, der 1978 von der Führung um Deng Xiaoping initiiert wurde, hat in den letzten zwei Jahrzehnten eine ökonomische Eigendynamik entwickelt, welche die schon vorhandenen regionalen und

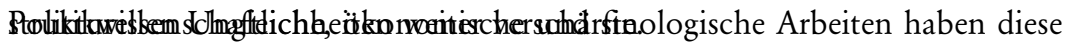
von der chinesischen Reformpolitik ausgelösten Transformationsprozesse von unterschiedlichen Perspektiven aus beobachtet und sind zu teilweise entgegengesetzten Prognosen und Beurteilungen gekommen. Zweifellos haben die ökonomischen Reformen ein wirtschaftliches Wachstum und eine Anhebung des Lebensstandards für zumindest einen Teil der Bevölkerung bewirkt. Jedoch hat der teilweise Rückzug des Staates aus den früheren sozialen Verpflichtungen tiefgreifende Veränderungen der Sozialstruktur zur Folge. Ungeachtet des Führungsanspruchs der Partei und des Festhaltens am sozialistischen System wird der wirtschaftliche Kurs nicht auf allen Ebenen politisch gesteuert. Diesen Entwicklungen soll Rechnung getragen werden, indem die unterschiedlichen Entwicklungsstrategien und ihre sozialen Folgen differenziert betrachtet werden.

\section{Die Komplexität weiblicher Realität}

In dem folgenden, von der „Komplexität der Realität“ (Leutner/Spakowski 1997: 125) ausgehenden Überblick soll die Situation chinesischer Frauen in 
Abhängigkeit von den regionalen Voraussetzungen, der Stadt-Land-Teilung und der unterschiedlichen Ausgangslage im Transformationsprozeß zu beschrieben werden. In den neueren Arbeiten, die sich mit den Geschlechterbeziehungen und der sozialen Stellung von Frauen in China befassen, wird ein differenzierter Forschungsansatz vertreten (vgl. Gilmartin et al. 1994, Leutner/Spakowski 1997, West et al. 1999). Es wird gefordert, Frauen nicht aus einer hegemonialen, eingeengten Sicht als bloße Opfer und Verliererinnen sozioökonomischer Entwicklungen zu betrachten, vielmehr soll ihre Rolle als Subjekte gesellschaftlicher Umstrukturierungsprozesse differenziert analysiert werden. Diese Ansätze der Genderforschung beziehen sowohl die historischen Bedingungen, als auch die sozialen Erfahrungen chinesischer Frauen mit ein, indem sie wie West u.a. in Feldstudien die Zusammenhänge zwischen patriarchalen Strukturen und Erwerbstätigkeit untersuchen oder auf das Verhältnis von Autonomie und staatlicher Lenkung eingehen. Die folgende Darstellung beschreibt unter Berücksichtigung der Kategorie Gender die komplexen Zusammenhänge im gegenwärtigen sozialen Differenzierungsprozeß der VR China und verknüpft sie mit den sozialen Bestimmungsfaktoren wie regionale Zugehörigkeit, Bildung und Alter. Dabei wird deutlich, daß Aussagen über die Auswirkungen der Transformationsprozesse auf die Situation der chinesischen Frauen nicht möglich sind, da eine Vielzahl an Variablen frauenpolitische Strategien behindern oder befördern können.

\section{Transformationsprozesse im ländlichen Raum}

Bis zu Beginn der achtziger Jahre gab es in China eine klare Trennung zwischen der städtischen und der ländlichen Gesellschaft. Letztere macht noch heute 70\% der Gesamtbevölkerung aus. Das System der Haushaltsregistrierung verhindert den Zuzug in die Stadt und schafft ungleiche Voraussetzungen für die Teilhabe am gesellschaftlichen Wachstum. Frauen aus dörflichen Gemeinden und Kreisstädten besitzen geringere Chancen zur Erwerbstätigkeit außerhalb des landwirtschaftlichen Sektors, weniger Bildungsmöglichkeiten und einen niedrigeren Lebensstandard als Frauen mit städtischem Wohnrecht. Letztere sind seit Gründung der Volksrepublik (1949) zu einem hohen Prozentsatz erwerbstätig. Durch die Einbindung in kollektive Produktionsprozesse in der Landwirtschaft und fehlende ökonomische Absicherung durch eigene Erwerbstätigkeit haben bäuerliche Frauen weniger Möglichkeiten der sozialen Eigenständigkeit und unterliegen stärker patriarchalen Familienstrukturen. Mit der Umstrukturierung der zentralen Planwirtschaft in eine diversifizierte und marktorientierte Ökonomie geht seit den 80er Jahren die Auflösung der ländlichen Kollektivproduktion und die Rückkehr zur familiären agrarischen Bewirtschaftung einher. Ein zentrales Ziel der wirtschaftlichen Reformen war die Steigerung der stagnierenden agrarischen Produktion und 
die Erhöhung des allgemeinen Lebensstandards. Die eigenverantwortliche Bewirtschaftung des Agrarlandes durch die bäuerlichen Haushalte legt für die Produzenten eine Abgabequote des Ernteertrags an den Staat fest. Sie fungiert als eine Art Landwirtschaftssteuer. Nach offiziellen Angaben hatten Ende 1983 bereits über 97\% aller ländlichen Haushalte das familiäre Selbstverantwortungssystem übernommen (Zhang 1993: 47). Mit der Reprivatisierung des landwirtschaftlichen Sektors wird so ein Anreiz zu höherer Produktivität geschaffen. Da der Anbau nicht mehr an den staatlichen Wirtschaftsplan gebunden ist, können die Familien auch über die Art der Anbauprodukte selbst entscheiden. Die meisten Haushalte entscheiden sich für einen Anbau gewinnbringender Produkte, wie spezielle Obst- und Gemüsesorten. Dieser ist allerdings an lokale Voraussetzungen gebunden und führt in manchen Gebieten zu einer rücksichtslosen ökologischen Ausbeutung des Bodens.

Die Entscheidungsfreiheit über die Produktion und die Unabhängigkeit von zentralen Vorgaben haben zu einer allgemeinen Anhebung des Lebensstandards der ländlichen Bevölkerung beigetragen, die sich jedoch in unterschiedlichem Maß auf Männer und Frauen auswirkt. Die Dezentralisierung und Rückführung der landwirtschaftlichen Produktion auf die bäuerlichen Haushalte verstärkt die Einbindung der Frauen in die Familie. Eine fehlende Alterssicherung auf dem Land und der Einsatz männlicher Arbeitskraft bei der Feldarbeit setzen Frauen dem Druck patriarchaler Wertvorstellungen aus. Die auf der Familienökonomie basierende Bewirtschaftung hat die traditionellen Erwartungen nach männlichen Nachkommen verstärkt. Bei einer Befragung von Frauen aus Dörfern in der wirtschaftlich entwickelteren Provinz Jiangsu und in einem weniger entwickelten ländlichen Raum der Provinz Sichuan, stellte die Forscherin einen übereinstimmenden Wunsch nach einer geringen Kinderzahl fest, wobei eine klare Präferenz für zwei Kinder deutlich wurde. Bei der Entscheidung für die Ein-Kind-Familie dominierte jedoch der Wunsch nach einem Sohn, in der geringer entwickelten Region war der Prozentsatz bei den Frauen höher (Wu 1999: 140).

Andererseits geben die veränderten Produktionsformen den Frauen einen größeren Spielraum für eigenständige Entscheidungen und erweiterte Erwerbsmöglichkeiten. Seit der Aufhebung der Lebensmittelrationierung und der Freigabe der Preise sind in den Städten und in deren Umkreis eine Vielzahl an „freien Märkten“ entstanden, die vorwiegend von Frauen betrieben werden. Mit der Liberalisierung des Wirtschaftssystems ist auch der zeitlich begrenzte Zuzug zur Arbeitsaufnahme in den Großstädten (Kreisstädte zählen zum ländlichen Raum) möglich. In den 90er Jahren sind enorme Migrationsbewegungen zu beobachten, vor allem von jungen Männern auf der Suche nach Erwerbsmöglichkeiten in den industriell höher entwickelten Küstenregionen. Gleichzeitig gibt es durch den gestiegenen Lebensstandard der Städter eine größere Nachfrage nach Agrarprodukten, die nach den Wettbewerbsge- 
setzen des freien Handels erhältlich sind. Mit den Einnahmen aus dem Verkauf sind ländliche Kollektiv- und Privatbetriebe im Bereich der Nahrungsmittelverarbeitung und im Dienstleistungssektor entstanden. Durch den gestiegenen Wohlstand wurde es möglich eine Vielzahl an kleinen Läden, Garküchen und Friseurgeschäfte als Kollektiv- oder Privatunternehmen zu gründen. Die Erwerbsmöglichkeiten außerhalb der Landwirtschaft haben die Eigeninitiative und das Selbstwertgefühl besonders der jüngeren Frauen gestärkt.

Chinesische Feldstudien betonen die positiven Auswirkungen der Reformpolitik im Hinblick auf den Freiraum für individuelle Entscheidungen im Vergleich zu den Einschränkungen durch die Vorgaben von Staat und Partei in den Volkskommunen (Zhang 1999: 51). Zwar hatte die Kollektivierung der Landwirtschaft die Frauen in den Produktionsprozeß eingebunden und die politische Mobilisierung durch Partei und Frauenorganisationen das gesellschaftspolitische Bewußtsein gefördert, doch waren die Grundkonzeptionen im Geschlechterverhältnis auf Ungleichheit aufgebaut. Die Entlohnung in den Volkskommunen nach Arbeitspunkten, die abgestuft nach Alter und Geschlecht festgelegt wurden, führten dazu, daß die Arbeitsleistung von Frauen im allgemeinen 20\% niedriger als die der Männer eingestuft wurden (Zhang 1999: 50). Hinzu kam, daß die Auszahlung des Lohns an die Leitung der Arbeitsbrigade erfolgte. Die aber war überwiegend von Männern besetzt. Somit blieben die Frauen wirtschaftlich und sozial weiterhin den patriarchalen Strukturen unterworfen und gleichzeitig verhinderten die staatlichen Planvorgaben autonome Entscheidungen.

Ein wichtiges Kriterium zur Beurteilung weiblicher Gleichberechtigung wird in der Geschlechterforschung dem Faktor Autonomie beigemessen (Zhang 1999: 49). Die starre Einbindung in kollektive Arbeitsprozesse ließ den Frauen wenig Chancen zu zusätzlicher Erwerbsmöglichkeit wie beispielsweise Kleintierhaltung oder Herstellung lokaler kunsthandwerklicher Produkte. Das restriktive städtische Zuzugsverbot, das selbst bei Heirat kein Wohnrecht gewährt, schränkt die soziale und geographische Mobilität der Landbevölkerung ein. Die Festsetzung der Preise von subventionierten Nahrungsmitteln und die künstlichen Niedrigpreise für Alltagsgüter bei steigenden Löhnen städtischer Arbeiter vergrößerten die Stadt-Land-Diskrepanz. Ein Wechsel vom Land zur Stadt ist nur in einem begrenzten Zeitraum möglich, und besonders Frauen bieten sich kaum Chancen auf Arbeit im Industriebereich außerhalb des ländlichen Sektors, wo das Lohnniveau höher ist und in den Staatsunternehmen soziale Sicherheitsgarantien gelten.

Aus chinesischer Sicht wurde die weibliche Erwerbstätigkeit lange Zeit als Grundlage für die Befreiung der Frauen angesehen, jedoch bildeten die Rückständigkeit der stagnierenden Produktion, die geringe ländliche Industrialisierung und der Mangel an sozialen Voraussetzungen (Kindergärten, Bildungs- 
einrichtungen, ärztliche Versorgung, Haushaltsgeräte) kaum eine Grundlage für eine Steigerung des Lebensstandards und eine selbstbestimmte Lebensplanung. Die Einführung des familiären Verantwortungssystems hat - nach Regionen und Alter differenziert - den Frauen im ländlichen Raum zusammen mit der Entwicklung des leichtindustriellen Sektors ökonomische und soziale Vorteile gebracht. Die Förderung einer Vielzahl von Kleinbetrieben in den stadtnahen Gebieten haben in den Jahren 1978 bis 1988 die Erwerbsmöglichkeiten außerhalb der Landwirtschaft erhöht und zu großen Wanderungsbewegungen innerhalb des Landes geführt, auch wenn die Mobilität auf dem Land weiterhin eingeschränkt ist. Neben den staatlichen Beschränkungen wirken sich hier die lokalen Netzwerke aus, die sowohl auf familiären Strukturen wie politischen Beziehungen gründen. Christiansen sieht daher den größeren Effekt der Wirtschaftsreformen in dem Transfer aus landwirtschaftlicher Arbeit in „neue“ Erwerbsfelder (Christiansen 1992: 90). 1987 lag der Anteil von weiblicher Erwerbstätigkeit in ländlichen Unternehmen bei 35\%, 1990 betrug er bereits 42\% (Leutner/Spakowski 1997: 136).

Zhang, die 1994 in einer Feldstudie Frauen in einem nordchinesischen Dorf in der Nähe von Tianjin interviewt hat, nennt als positive Auswirkungen der Reformpolitk die größere individuelle und finanzielle Unabhängigkeit. Zur Zeit ihres Aufenthaltes gab es im Umkreis von Dongdatun drei Bekleidungsunternehmen und eine Textilfabrik, die sich im Besitz des Kreises und der Gemeinde befanden, ferner mehrere Kleinbetriebe unter privater oder kollektiver Leitung, die viele Frauen beschäftigten (Zhang 1999: 59). Bei ihrer Untersuchung zu den Veränderungen im sozialen Status der Frauen durch die Wirtschaftsreformen kam sie zu der Einschätzung: „Die Zunahme an Chancen zur Erwerbstätigkeit und die zusätzlichen Arbeitsmöglichkeiten außer Haus haben in einem gewissen Umfang ihre Position innerhalb der Familien erhöht und machen sie unabhängiger und mutiger sich der elterlichen oder patriarchalen Kontrolle über ihr Leben zu widersetzen; gleichzeitig haben sie ein größeres Vertrauen, ihre Vorstellungen aus eigener Kraft zu erreichen“ (Zhang 1999: 56). Es sind in erster Linie junge und unverheiratete Frauen, die in den Betrieben der Sonderwirtschaftszonen (SWZ) sowie Fabriken und Manufakturen der Textil-, Bekleidungs-, Papierverarbeitungs- und Nahrungsmittelindustrie als ungelernte Arbeiterinnen beschäftigt werden. Frauen aus den ländlichen Regionen nutzen die Chancen größerer Mobilität, um Zutritt zum Arbeitsmarkt in den exportorientierten, multinationalen Unternehmen in den küstennahen Regionen zu erhalten. „In den Betrieben der Leichtindustrie der Sonderwirtschaftszone Shenzhen beträgt das Verhältnis der weiblichen Aushilfsarbeiter zu ihren männlichen Kollegen bereits 2:1“ (Leutner/Spakowski: 135). Die Arbeitsbedingungen liegen weit unter den internationalen Normen, Sicherheits- und Gesundheitsvorschriften werden nicht eingehalten und $80 \%$ der Arbeiterinnen leiden unter Berufskrankheiten. 
Im Vergleich zu den Erwerbsmöglichkeiten in den ländlichen Industriebetrieben sind die Löhne höher, und die Arbeiterinnen können durch ihre ökonomische Selbständigkeit ihren sozialen Status erhöhen. Es läßt sich die Entstehung einer neuen sozialen Schicht erkennen, die zwischen der vorwiegend auf Subsistenzwirtschaft basierenden Landbevölkerung und den in den staatlichen städtischen Betrieben Angestellten einzuordnen ist. Aus chinesischer Sicht wird diese Teilhabe an industrieller Erwerbsarbeit als weiblicher Beitrag des Modernisierungskonzepts angesehen und als ein frauenpolitisches Entwicklungsmodell propagiert: „Sie sind die Heldinnen im Wandel des Frauenstatus im ländlichen Raum. Man kann konstatieren, daß die Industrialisierung ein Weg für die Modernisierung auf dem Land ist, denn diese Gruppen von Frauen repräsentieren die Richtung, in welche der Status der Frauen vom Land sich bewegen wird" (Gao 1994: 85). Neben Arbeiten in Niedriglohngruppen im industriellen Sektor hat die Migrationsentwicklung zu einer vermehrten Arbeitsaufnahme im Dienstleistungsbereich in den Städten geführt. Ebenso wie in den Fabriken besetzen auch hier die Frauen lediglich die Positionen, die wegen schlechter Bezahlung, mangelnder Arbeitsplatzsicherheit oder geringer Zukunftschancen von den städtischen Erwerbstätigen (Männern wie Frauen) ausgeschlagen werden (Huang 1999: 95). Junge, ledige Frauen aus den wirtschaftlich unterentwickelten Regionen arbeiten in privaten Dienstleistungsbetrieben wie Kantinen, Hotels und Läden. Von 1984 bis 1988 hat die Zahl der Beschäftigten in diesem Sektor um das Vierfache zugenommen, wovon $80 \%$ weibliche Beschäftigte - und mehr als die Hälfte vom Lande - waren (Huang 1999: 96). Da die Besitzer privater Unternehmen gehalten sind, Personal mit städtischem Wohnrecht bevorzugt einzustellen, werden Frauen vom Land vorwiegend für die schlechtbezahlten, schmutzigen und körperlich schweren Arbeiten eingestellt, zumal sie oft nicht den Bildungsgrad der städtischen Angestellten besitzen.

Ein weiteres Arbeitsfeld, das von Frauen aus den ländlichen Gebieten eingenommen wird, ist die Hausarbeit in Privathaushalten. 1989 waren 3 Millionen Frauen vom Land als Haus- und Kindermädchen in städtischen Haushalten registriert (Huang 1999: 95). Ein großer Teil von ihnen hat weder soziale Sicherheit noch eine Mindestlohngarantie. Meist kehren sie zur Heirat in die heimatlichen Kreise und Gemeinden wieder zurück und nutzen das ersparte Geld, sofern es nicht dem Familieneinkommen diente, im tertiären Sektor für das Betreiben von Verkaufsständen, Garküchen oder Friseurgeschäften. Je nach regionalen Voraussetzungen erwirtschaften sie durch Kleintier-, Fischoder Perlenzucht ein eigenes Einkommen. Daher sind die Auswirkungen der Reform- und Wirtschaftspolitik für Frauen aus den ländlichen Regionen unterschiedlich zu bewerten. Sie sind zwar in den industriellen Entwicklungsprozeß und in die Ausweitung des Dienstleistungssektors einbezogen, jedoch im Vergleich zu den männlichen Arbeitskräften und den Frauen 
aus den urbanen Zentren zu schlechteren Bedingungen. In manchen ländlichen Regionen können sie das Vakuum, das durch die Migration männlicher Arbeitskräfte in die industrialisierten Zentren entsteht, durch Erwerbsmöglichkeiten im Umfeld der landwirtschaftlichen Produktion füllen. In diesen Regionen können auch ältere Frauen ohne oder mit geringer Schulbildung eine Erwerbstätigkeit finden, die jedoch bei nicht diversifizierten landwirtschaftlichen Tätigkeiten bis zu 38\% schlechter bezahlt wird als bei diversifizierter agrarischer Tätigkeit (Leutner/Spakowski 1997: 138). Mit der Abwanderung der Männer vom Land stellen Frauen fast 50\% der ländlichen Arbeitskräfte, in einigen Gegenden sogar bis zu 70\% (Zhang 1999). Es wird daher bereits teilweise von einer „Feminisierung“ des Agrarbereichs gesprochen, der durch niedrigeren Lebensstandard, unsicheren Profit und fehlende soziale Sicherungssysteme (Krankenversicherung, Alters- und Gesundheitsversorgung) gekennzeichnet ist.

\section{Privatisierung, Erwerbstätigkeit und soziale Folgen in den urbanen Zentren}

Seit der Gründung der VR China ist die Einbeziehung von Frauen in den Produktionsprozeß eines der zentralen Ziele der Parteiführung gewesen, um die Gleichberechtigung und den sozialen Status in Familie und Gesellschaft voranzutreiben. In der Praxis wurde das Recht auf gleiche Chancen zur Erwerbsarbeit durch die wirtschaftlichen Notwendigkeiten beim Aufbau des Landes bestimmt. Waren vor 1949 in der Stadt Nanjing noch über 70\% der Frauen ohne bezahlte Arbeit, so waren zwischen 1950 und 1966 bereits fast 71\% der verheirateten Frauen berufstätig. Bis 1986 galt in den Staatsunternehmen das Prinzip der lebenslangen Beschäftigung, d.h. der Unkündbarkeit bei festgelegten Löhnen. Dieser sichere Arbeitsplatz bedeutete gleichzeitig auch soziale Absicherung durch die Vergabe von Wohnraum sowie Gesundheits- und Altersvorsorge, ließ aber auch kaum einen Wechsel zu einem anderen Betrieb zu. In der Verfassung von 1982 ist ausdrücklich die Pflicht des Staates festgeschrieben, die Rechte und Interessen von Frauen zu schützen, Lohngleichheit zu sichern und die Heranbildung von weiblichen Führungskräften zu fördern. Statistische Daten von 1987 weisen für Chinesinnen aus den Städten international die höchste Rate an erwerbstätigen Frauen auf, wonach 9 von 10 Frauen einer bezahlten Arbeit nachgehen (Bauer et al. 1999: 350). Die sozialen und arbeitsrechtlichen Schutzbestimmungen gelten jedoch vor allem für die staatlichen Einrichtungen. 1988 betrug der Anteil an Frauen in staatlichen Betrieben ca. 33\%, in Kollektivbetrieben 47\% und in Betrieben mit anderen Eigentumsformen 50\% (Borchard 1992: 192). Allerdings sind die Leistungen in den Kollektiv- und Privatunternehmen von dem wirtschaftlichen Erfolg abhängig, Löhne und Prämien sind variabel. Waren bis 1986 
Vollbeschäftigung und ein sicherer Arbeitsplatz mit sozialen Sicherheiten die Grundprinzipien staatlicher Politik, so wurde mit der Reform des Arbeitsvertragssystems ein frühkapitalistischer Arbeitsmarkt geschaffen. Die Wirtschaftsreformen haben den Druck auf die Betriebe, Profite zu maximieren, erhöht und zu Rationalisierungen und Entlassungen sowie Umsetzungen des ,überschüssigen" Personals geführt. Diese Entwicklungen zeigen jedoch deutlich unterschiedliche Wirkungen auf die Geschlechter, die zusätzlich nach Industriezweigen differieren. Für die VR China ist eine klare Hierarchie im industriellen Sektor zu verzeichnen. Die höchsten Löhne werden in den Bereichen der Schwer- und Ölindustrie sowie im Bergbau mit einem hohen Anteil an männlichen Lohnarbeitern gezahlt. Auch in der nächst höheren Gruppe, wie Maschinenbau, Bauwesen und chemischer Industrie, ist der Anteil an Frauen gering. Lediglich in dem am niedrigsten eingestuften Produktionssektor zeigt sich ein hoher Anteil an weiblichen Erwerbstätigen: Textil-, Porzellan-, Tabakund Nahrungsmittelindustrie (Bauer 1999: 359).

Dieser segmentierte Arbeitsmarkt findet sich ebenso in den entwickelten Industrieländern, hat jedoch in China noch zusätzliche Dimensionen. Die Konzentration von Frauen in der leichtindustriellen Produktion der Textilund Bekleidungsindustrie, in der 8 von 10 Beschäftigten Frauen sind, ist von geringen arbeitsrechtlichen Sicherheiten und frühkapitalistischen Arbeitsbedingungen gekennzeichnet. Der im Vergleich zu gleichaltrigen Männern höhere Anteil an jungen Frauen zwischen 15 und 19 Jahren in den städtischen Betrieben weist auf den geringeren Anteil an weiblichen Jugendlichen in der Ausbildung hin. Mit der Entstehung eines „zweigleisigen Modells“ von Planund Marktwirtschaft gerieten die Staatsbetriebe unter den Druck des marktwirtschaftlichen Wettbewerbs, unrentable Betriebe wurden privatisiert und innerbetrieblich wurden Umstrukturierungen vorgenommen. Da in den staatlichen Betrieben Entlassungen nicht möglich waren, wurde das „überschüssige Personal“ umgesetzt; dies war mit geringeren Löhnen und Prämien verbunden. Diese Maßnahmen treffen vor allem Frauen, die aufgefordert werden, früher in den Ruhestand zu gehen oder längeren Erziehungsurlaub in Anspruch zu nehmen. Nach einer Studie des Nanjinger Frauenverbands waren knapp 72\% der Entlassenen in der Transportbranche Frauen (Borchard 1992: 207). Die Umstrukturierungen in den städtischen Unternehmen sind vor allem von der jeweiligen Branche, dem vorhandenen Kapital und der Chance, wie sich der Betrieb in dem marktwirtschaftlichen Wettbewerb positionieren kann, abhängig.

Da Frauen durch die gesetzlichen Schutzbestimmung (Schwangerschaft, Stillzeit etc.) für die Unternehmen zu einem höheren Kostenfaktor gegenüber den männlichen Arbeitern geworden sind, werden sie trotz beruflicher Qualifikation und höherem Bildungsgrad auch bei Neueinstellungen benachteiligt. Vor allem Frauen mit Kleinkindern und ältere Frauen, die bereits mit 50 Jah- 
ren in den Ruhestand geschickt werden, wird die „Rückkehr an den Herd“ geraten. Der Ausschluß aus der Erwerbsarbeit bedeutet soziale Einschnitte, da lediglich 50-75\% des Grundgehaltes weiter gezahlt werden (Woo 1999: 285). Frauen mit hoher Qualifikation gelingt es zunehmend schwerer eine adäquate Arbeit mit gesichertern sozialen Leistungen zu erhalten. Sie müssen sowohl mit den gut ausgebildeten Männern konkurrieren, die in die Privatunternehmen drängen, als auch mit den Frauen aus ländlichen Gebieten, die in den leichtindustriellen Sektoren zu Niedrigstlöhnen arbeiten. Für junge Frauen bleibt vor allem der Dienstleistungssektor, wo in den Bereichen Tourismus, Hotel- und Gaststättengewerbe neue Arbeitsplätze entstehen.

Es sind die Bereiche Mode, Werbung und Unterhaltung, in denen sich einige Frauen selbständig gemacht und in den westlichen Medien Aufmerksamkeit erhalten haben. Bei einer genaueren Analyse zeigt sich jedoch, daß diese Möglichkeiten vor allem denen vorbehalten bleiben, die auf ein Netzwerk politischer Verbindungen oder auf Kapital aus Joint-VentureUnternehmungen zurückgreifen können. Zusammenfassend läßt sich sagen, daß sich die ökonomischen Transformationsprozesse in unterschiedlicher Weise auf die Frauen auswirken. In den ländlichen Gebieten bieten sich für Frauen einige, wenn auch begrenzte, Möglichkeiten zu mehr Eigenständigkeit, in den Städten ist die Situation dagegen von einem Verdrängungswettbewerb gekennzeichnet.

\section{Frauenpolitische Strategien und politische Partizipation}

Seit Beginn der Reformpolitik sind die wirtschaftlichen und sozialen Auswirkungen auf die Situation der Frauen ein zentrales Thema frauenpolitischer Aktivitäten. Im Zentrum stand dabei zunächst die Frage, inwieweit Frauen durch die wirtschaftlichen Umstrukturierungen in den produktiven Bereich einbezogen werden. Angeregt durch den Austausch mit Frauen der internationalen Frauenbewegung während der Weltfrauenkonfrenz 1995 in Peking und durch Ansätze der sozialwissenschaftlichen Genderforschung haben sich die Forschungsanstrengungen auf differenzierte Fragen der ungleichen Voraussetzungen und Folgen des Transformationsprozesses verlagert.

Chinesische Forscherinnen befassen sich u.a. mit dem niedrigen Status von Frauenarbeit, der durch den hohen Anteil berufstätiger Frauen verdeckt wurde. In ihrer empirischen Studie, die Daten von 1987 verwendet, setzen sich Bauer et al. mit der sozialen Lage von städtischen Frauen auseinander, da der Anteil weiblicher Erwerbstätiger in den urbanen Zentren seit Gründung der VR China besonders hoch war. Sie konstatieren die geringeren Bildungschancen, aber auch das Abdrängen von Frauen aus dem Arbeitsmarkt. Letzteres wird von Kampagnen zur Rückkehr an den Herd flankiert. Um die Chancen für Frauen aus ländlichen Gebieten unter den neuen Wettbewerbsbedingun- 
gen zu erhöhen, wurden seit 1989 spezielle Förderprogramme initiiert. Sie sollen die Analphabetenrate reduzieren, Kenntnisse der neuen Technologien vermitteln und das Selbstbewußtsein stärken (Zhang 1999). Das staatliche Programm wurde unter dem Slogan „shuangxue, shuangbi“ (Doppelstudium, doppelter Wettbewerb) vom Nationalen Frauenverband umgesetzt. Als Folge wurden verschiedene Bildungsinitiativen für Existenzgründungen entwickelt. Das Programm umfaßte Vermittlung von Wissen u.a. zu Kreditwesen, Marketing und Technologieeinsatz und wurde mit Aktionen zur Aufforstung und zum Umweltschutz gekoppelt. Nach chinesischen Angaben haben in dem Zeitraum von 1989 bis 1994120 Millionen Landfrauen an diesen Programmen teilgenommen. 20 Millionen Frauen konnten alphabetisiert werden. Auch wenn die amtlichen Angaben nicht unbedingt verläßlich sind und auch der Weg der eingesetzten Gelder nicht genau verfolgt werden konnte, so verweist Zhang dennoch darauf, daß die Regierung bemüht ist, „Frauen durch die Diversifikation der ländlichen Ökonomie in die ländlichen Entwicklungsprogramme zu integrieren und sie zur Teilnahme an diesen Programmen zu bestärken" (Zhang 1999: 59).

Trotz der Kritik an den Folgen des Transformationsprozesses verlangen chinesische Frauenrechtlerinnen in ihrer Mehrheit nicht die Rücknahme der Reformpolitik. Sie setzen auf eine Mischung von Eigeninitiative und staatlicher Frauenpolitik und plädieren dafür, ein grundlegendes Sozialsystem zu schaffen, das für die städtische und ländliche Bevölkerung gleichermaßen geeignet ist. Auch wenn die Situation der Frauen von einer Ungleichzeitigkeit innerhalb der gesellschaftlichen Transformationsprozesse gekennzeichnet ist, so lassen sich doch gewisse Gemeinsamkeiten erkennen: ungleiche Bezahlung für gleiche Arbeit, Verlust sozialer Sicherheiten, geringere Chancen in den besser bezahlten Sektoren und eine grundlegende Benachteiligung aufgrund des Geschlechts auch bei Einbeziehung in den Produktionsprozeß.

Vor diesem Hintergrund stellt Chinas exponierteste Frauenforscherin Li Xiaojiang die Frage nach der Berechtigung des Prinzips „Gleichheit“ von Frau und Mann. Erst auf der Grundlage rechtlicher Gleichberechtigung und einer wirklich sozialen und entwickelten Gesellschaft - auch wenn sie von männlichen Prinzipien bestimmt ist - läßt sich für sie tatsächlich die „Befreiung der Frauen" entwickeln. In einer noch weitgehend unterentwickelten Gesellschaft wie China tragen die Frauen nach wie vor die größeren Lasten. Jedoch haben sie durch die bisher erreichte soziale Ausgangsposition bessere Chancen, nunmehr ein kollektives Bewußtsein für ihre spezifischen Bedürfnisse und ein weibliches Selbstbewußtsein zu entwickeln. Nach den Worten von Li könnten nun mit der wirtschaftlichen Reform die „neuen Frauenthemen wie Gräser im Frühlingsregen sprießen.“ 


\section{Literatur}

Bauer, John/Wang, Feng/Riley, Nancy E./Zhao, Xiaohua (1992): Gender Inequality in Urban China. Education and Employment, in: Modern China, Vol. 18, Nr.3, 333-370.

Borchard, Dagmar (1992): Auswirkungen der Arbeitsgesetzgebung der 80er Jahre auf die Frauenarbeit, in: Frauenstudien, 123-130.

Christiansen, Flemming (1992): „Market Transition“ in China. The Case of the Jiangsu Labor Market, 1978-1990, in: Modern China, Vol. 18, Nr.1, 72-93.

Gao, Xiaoxian (1994) China's Modernization and Changes in the Social Status of Rural Women, in: Engendering China, 80-97.

Gilmartin, Christina K./Hershatter, Gail/Rofel, Lisa/White, Tyrene eds. (1999): Engendering China. Women, Culture and the State. New York.

Huang, Xiyi (1999): Divided Gender, Divided Women. State Policy and the Labour Market, in: Women of China, 90-107.

Leutner, Mechthild/Nicola Spakowski (1997): Die „Komplexität der Realität“. Chancen und Rückschritte von Frauen im Transformatiosprozeß Chinas, in: Frauen-Los!? Politische Partizipation von Frauen in Ostasien, hg. von Heberer/Katharina Vogel, Hamburg, 125-171.

Li, Xiaojiang (1994): Economic Reform and the Awakening of Chinese Women's Collective Consciousness, in: Engendering China, 360-381.

Li, Xiaojiang (1994): Ein Rückblick auf die Frauenbewegung der neuen Zeit, in: Newsletter "Frauen und China", H. 6, 32-35

Sandschneider, Eberhard (1996): Perspektiven chinesischer Reformpolitik. Das Dilemma zwischen Stabilität und Transformation, in: Berliner China-Hefte, H.11, 3-10.

Sausmiskat, Nora (1995): Li Xiaojiang und ihre feministische Theorie, in: Frauenforschung in China. Hg. von Heike Frick, Mechthild Leutner, Nicola Spakowski, 111-118.

Spakowski, Nicola (1994): Wohin geht die chinesische Frauenbewegung?, in: Newsletter "Frauen und China", H. 6, 36-38.

West, Jackie/Zhao, Minghua/Chang Xiangqun/Cheng, Yuan eds. (1999): Women of China. Economic and Social Transformation. New York.

Woo, Margaret Y. K. (1994): Chinese Women Workers. The Delicate Balance between Protection and Equality, in: Engendering China, 279-295.

Zhang, Heather Xiaoquan (1999): Understanding Changes in Women's Status in the Context of the Recent Rural Reform, in: Women of China, 45-66

Zhang, Minjie (1993): Der Wandel der Stellung der chinesischen Frau in der Familie, in: Newsletter „Frauen und China“ H. 5, 36-51.

Zheng, Bijun (1996):: Women’s Movement and Modernization, in: Berliner China-Hefte, H.10, 3-10. 